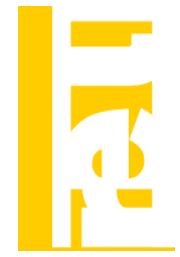

\title{
DESEMPLEO Y PRESTACIONES EN LA OBRA DE LUIS TOHARIA
}

\author{
José María Arranz, Carlos García Serrano \\ Universidad de Alcalá
}

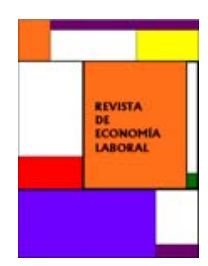

\section{Resumen}

Este artículo se centra en examinar varios trabajos escritos por Luis Toharia (la mayoría en colaboración con investigadores de diversas universidades) que tienen que ver con la eficiencia del sistema de protección por desempleo, en particular el grado en que las prestaciones por desempleo influyen en los procesos de búsqueda y salida hacia el empleo, así como otros aspectos relacionados, tales como la cobertura del sistema y su grado de generosidad.

Palabras clave: Prestaciones por desempleo, salida del paro, mercado de trabajo español. Clasificación JEL: J64, J65.

\begin{abstract}
This article focuses on the examination of various works written by Luis Toharia (the majority joint with researchers from diverse universities) which have to do with the efficiency of the unemployment compensation system, in particular the degree to which unemployment benefits influence the processes of search and exit to a job, and other related issues, such as the take-up of the system and its degree of generosity.

Key words: Unemployment benefits, exit from unemployment, Spanish labour market.
\end{abstract}

JEL Classification: J64, J65.

\section{Introducción}

Luis Toharia siempre dedicó una gran parte de sus esfuerzos al estudio de las causas y las consecuencias del desempleo en el mercado de trabajo español ${ }^{1}$. Dentro de su brillante carrera investigadora estuvo preocupado por la mejora de la comprensión de los vínculos del Sistema de Protección por Desempleo (SPD) con los procesos de búsqueda de empleo y salida del paro por parte de los trabajadores desempleados. Su inigualable capacidad como investigador interdisciplinar ha permitido conseguir que sus resultados y reflexiones obtenidos en estos trabajos se hayan convertido en la base para muchos investigadores, entre los que nos encontramos nosotros dos. En este artículo examinamos varios trabajos

\footnotetext{
${ }^{1}$ Véase, por ejemplo, Toharia y Fina (1987).
}

(C) Revista de Economía Laboral 
suyos (la mayoría de ellos escritos en colaboración con investigadores de diversas universidades) relacionados con la eficiencia del SPD, en particular el grado en que las prestaciones por desempleo influyen en los procesos de búsqueda y salida hacia el empleo, así como otros aspectos relacionados, tales como la cobertura del sistema o su grado de generosidad $^{2}$.

\section{Las prestaciones por desempleo y la búsqueda de empleo}

La teoría económica convencional predice que la percepción de prestaciones por desempleo supone un desincentivo a la búsqueda de empleo. Esta es la explicación teórica que se suele utilizar para explicar los resultados empíricos de que los perceptores de prestaciones por desempleo presentan una menor probabilidad de salida del paro en comparación con los no perceptores. Por tanto, el mecanismo implícito entre las prestaciones y la salida del paro sería la búsqueda de empleo, que se supone resulta influida por la percepción o no de prestaciones.

Hay que señalar que muy pocos trabajos han estudiado empíricamente esta cuestión en España, siendo Luis Toharia uno de los pocos autores que se ha dedicado a ello, publicando varios artículos con resultados relevantes. Así, en un artículo de $2003^{3}$, utilizando los microdatos de la Encuesta de Población Activa (EPA) para el período 19872003, analiza cuántos de los perceptores de prestaciones no buscan empleo efectivamente ( $\mathrm{y}$, por tanto, no son clasificados como parados sino como inactivos), teniendo en cuenta que el criterio básico para delimitar a los parados es que declaren estar buscando empleo. En particular, lleva a cabo un cruce de la pregunta sobre la situación con respecto al SPD (que se hace a todos los individuos) y la situación con respecto a la actividad (que se determina a partir de respuestas a preguntas anteriores). Sus resultados indican que el porcentaje de perceptores que son ocupados fue disminuyendo hasta alcanzar cifras relativamente pequeñas (4-5 por ciento entre los varones y 3-4 por ciento entre las mujeres en 1993-2003), mientras que el porcentaje de los que son inactivos siguió una tendencia creciente llegando a situarse en el 20-22 por ciento en el caso de los hombres y en el 30-34 por ciento en el de las mujeres en el período 19992003. Este colectivo de perceptores no buscadores clasificados como inactivos estaba formado fundamentalmente por personas con edades a

\footnotetext{
2 Toharia, L. (1997).

${ }^{3}$ Cantó y Toharia (2003).
} 
partir de $45 \mathrm{y}$, sobre todo, 55 años: en el caso de las mujeres, más de la mitad de las perceptoras de más de 55 años son inactivas, mientras que para los otros grupos de edad la cifra no llega al 30 por ciento.

Otro trabajo suyo publicado en el año $2000^{4}$ estudia el vínculo entre las prestaciones por desempleo y la búsqueda de empleo utilizando dos fuentes de datos: la EPA (segundo trimestre de 1998) y la Encuesta sobre las Condiciones Sociales de los Desempleados en España (CSDE) realizada por el CIS por encargo del INEM. Sus principales resultados se refieren a cuatro aspectos.

Primero, la clasificación de los individuos: algunos perceptores de prestaciones no buscan activamente empleo, pero la EPA no los considera parados sino inactivos. Segundo, la búsqueda activa: entre los parados inscritos en las oficinas públicas de empleo, todos aquellos que cobran prestaciones (independientemente de la duración de su último empleo) no presentan una probabilidad significativamente distinta de ser buscadores no intensos, es decir, que sólo buscan empleo mediante la inscripción en dichas oficinas (con la información de la EPA) y no hay diferencias significativas entre los individuos según que perciban o no prestaciones por desempleo en cuanto a la probabilidad de que hayan buscado activamente empleo durante los últimos tres meses (con los datos de la CSDE).

Este resultado es corroborado en otro artículo publicado en $2003^{5}$, en el que se aprovecha el cambio en la definición de desempleo aprobado por la Comisión Europea en septiembre de 2000 y recogido en la EPA española desde el primer trimestre de 2001. Esta nueva definición plantea un criterio más riguroso de búsqueda de empleo para que una persona pueda ser considerada parada. Su aplicación conlleva la distinción de un grupo de "buscadores pasivos" que dejan de ser parados y pasan a formar parte de la población inactiva. Esto plantea la posibilidad de determinar hasta qué punto la percepción de prestaciones por desempleo es una variable que incide en la probabilidad de ser un buscador pasivo, o sea, de estudiar si las prestaciones inhiben la búsqueda activa de empleo. El análisis realizado sugiere que las prestaciones por desempleo no afectan positivamente a la probabilidad de ser un buscador pasivo, es decir, no inhiben la búsqueda activa de empleo.

El tercer aspecto se refiere a la intensidad de la búsqueda: tampoco hay diferencias significativas entre perceptores y no perceptores en cuanto a la intensidad de búsqueda aproximada por el número de métodos utilizados. Y finalmente el cuarto tiene que ver con la disposición a aceptar

\footnotetext{
${ }^{4}$ García Mainar, y Toharia (2000).

${ }^{5}$ Garrido y Toharia (2003).
} 
un empleo: la percepción de PC incide negativamente en dicha disposición, lo que puede interpretarse como que la percepción eleva el salario de reserva de los parados. Los resultados indican que esto sucede en dos-tres de las cinco condiciones analizadas (aceptar un empleo a tiempo parcial, aceptar un salario inferior y, en cierta medida, aceptar una cualificación inferior).

\section{- Las prestaciones por desempleo y la salida del paro}

En los estudios que acabamos de revisar, los resultados más relevantes indican que las prestaciones no afectan a la probabilidad relativa de que los perceptores de prestaciones reciban ofertas de empleo (puesto que sus actividades de búsqueda no se ven afectadas por la percepción, en comparación con otros parados no perceptores) pero influyen negativamente en la probabilidad relativa de que las ofertas sean aceptadas (dado que la disponibilidad de los perceptores es menor que la de los no perceptores), por lo que en principio cabría pensar que la percepción de prestaciones por desempleo reduce la probabilidad de que los parados salgan de su situación y alarga la duración de los episodios de desempleo. Siempre dispuesto a desafiar la sabiduría convencional, Luis Toharia se plantea en otro conjunto de estudios comprobar empíricamente si, dados los resultados anteriores, las prestaciones inciden efectivamente en la probabilidad de salir del paro y encontrar un empleo.

Para ello, la información necesaria debería ser de naturaleza longitudinal. Este tipo de datos no eran habituales ni mucho menos en España a principios de los años noventa del siglo XX. Pero como Luis Toharia siempre ha mostrado interés por disponer de y explotar información relevante y novedosa para poder aplicar sus ideas, dedicó (mucho) tiempo a generar datos longitudinales y a desarrollar tres líneas de investigación utilizando este tipo de información.

La primera línea se basó en la utilización de los datos de los perceptores de prestaciones del Servicio Público de Empleo Estatal (Histórico del Sistema de Prestaciones, HSIPRE) a comienzos de los años 1990. La segunda línea de investigación relacionó las prestaciones y la salida del paro a partir de los datos de la EPA enlazada (panel rotante), que permite el seguimiento de los individuos durante seis trimestres consecutivos y que comenzó a ponerse a disposición de los investigadores en 1996. La tercera línea se llevó a cabo con la explotación de los datos de la Muestra Continua de Vidas Laborales (MCVL) disponible a partir de 2004.

Respecto a la primera línea de investigación, se inició con la realización de dos estudios encargados por el Banco de España y la 
Fundación Empresa Pública ${ }^{6}$ y se basó fundamentalmente en la utilización de la base de datos HSIPRE. Esta presentaba un problema: sólo se observaban perceptores de prestaciones, es decir, no existía un grupo de control con el que comparar la conducta de los perceptores. Sin embargo, tenía una gran ventaja: disponía de información tanto de la duración potencial (y efectiva) del período de derecho como de la cuantía de la prestación. Por tanto, podría emplearse para analizar cuestiones relacionadas con la posible influencia de la duración del derecho potencial y el nivel de la prestación sobre la salida del paro. La utilización de estos datos (los correspondientes al período 1984-1991) dio lugar a la publicación de varios trabajos en colaboración con un grupo de profesores de la Universidad de Alcalá: un capítulo de un libro en $1995^{7}$, un libro en $1996^{8}$ y un artículo en $1996^{9}$.

Algunos de los resultados obtenidos en estos trabajos son los siguientes. Primero, la duración del derecho resulta una variable significativa a la hora de explicar el proceso de salida (hacia el empleo) de los perceptores de prestaciones contributivas, sobre todo en el caso del grupo que tiene el máximo derecho legal de 24 meses, cuyas tasas de salida son claramente inferiores a las de los demás grupos. Segundo, en cuanto a la influencia del nivel de la prestación en relación con el salario, la tasa de sustitución no es significativa a la hora de explicar la salida hacia el empleo, excepto en el caso de los perceptores con derechos de corta duración. Tercero, la probabilidad de salida del paro es mucho mayor para los que entraron en el sistema de prestaciones procedentes de un contrato temporal en comparación con quienes perdieron un empleo indefinido. Finalmente, se detecta la existencia de un notable nivel de desempleo recurrente, es decir, individuos que salen del sistema y vuelven a entrar en él: en torno al 30 por ciento de los perceptores vuelven a aparecer en el sistema en un plazo de dos años, proporción que se eleva a más del 50 por ciento si el intervalo de tiempo se amplía a cuatro años.

Respecto a segunda línea de investigación, debe señalarse que la EPA enlazada dispone de información limitada de las prestaciones por desempleo, ya que se refiere solamente a si el individuo recibe prestaciones o no (aunque esto permite disponer de un grupo de control), pero no permite conocer el nivel de las prestaciones ni la duración del

${ }^{6}$ Los estudios son los siguientes: "Los perceptores de desempleo en España", financiado por el Banco de España (diciembre 1992-junio 1994); y "El sistema de protección por desempleo en España: situación y perspectivas”, encargado por la Fundación Empresa Pública (diciembre 1993-junio 1994).

${ }^{7}$ Cebrián et al. (1995).

${ }^{8}$ Cebrián et al. (1996a).

${ }^{9}$ Cebrián et al. (1996b). 
período de derecho ni distinguir entre prestaciones contributivas o asistenciales $^{10}$.

Evidentemente, la no consideración de estas variables en un análisis de la salida del paro puede llevar a la obtención de resultados incorrectos, ya que el colectivo de perceptores de prestaciones no es un colectivo homogéneo sino muy diverso, que recibe tipos de prestaciones distintas, con diferente duración y cuantía. Ya en 1998 Luis Toharia examina esta cuestión ${ }^{11}$ : considerando períodos de observación de un año en vez de trimestre a trimestre con al EPA enlazada, plantea una variable que combina percepción de prestaciones y períodos de derecho imputado (vía duración del empleo anterior). Los resultados que obtiene son los siguientes: tomando como referencia los parados que no cobran prestaciones y cuyo último empleo duró como máximo un año, los que sí cobran y su último empleo duró como máximo un año muestran una probabilidad algo mayor de salir hacia el empleo; los que sí cobran y su último empleo duró entre dos y cinco años no muestran diferencias significativas; y los que cobran y su último empleo duró seis años o más (lo que les generaría la duración máxima de prestaciones) tienen una probabilidad claramente menor de salir hacia el empleo (y lo mismo sucede con quienes no cobran y su último empleo duró dos o más años, y los que dejaron el empleo hace más de ocho años). Estos resultados sugieren que las prestaciones por desempleo ejercen una influencia negativa clara en la probabilidad de salida del paro de los trabajadores que estuvieron mucho tiempo en su anterior empleo, resultado coincidente con los de sus trabajos de 1995 y 1996 mencionados antes.

En su estudio de 2003 revisado más arriba, Luis Toharia también investigaba si la influencia de las prestaciones por desempleo en la probabilidad de salida hacia el empleo es sensible al período de análisis elegido, al método de identificación de las personas que han perdido su empleo y al horizonte temporal al que se refiere la salida del paro. Para ello, el período de análisis (1992-2003) se divide en dos subetapas (19921998 y 1999-2003); la identificación de los nuevos parados se realiza mediante dos métodos alternativos (seguir a las personas que están ocupadas en la primera entrevista y paradas en la segunda, para analizar

\footnotetext{
10 Este último aspecto es relevante, como han puesto de manifiesto por Toharia et al. (2006). Estos autores utilizan datos administrativos de parados registrados en dos meses sucesivos (diciembre de 2005 y enero de 2006) y encuentran que, aunque los perceptores de prestaciones muestran una probabilidad menor de salir al empleo que los no perceptores, este resultado se concentra entre quienes reciben la prestación asistencial y, sobre todo, entre los que reciben el subsidio de mayores de 52 años y otros (principalmente, renta activa de inserción).

11 Toharia (1998).
} 
a continuación su situación; o seguir a las personas que están desempleadas en la primera entrevista y dicen llevar menos de tres meses en su situación); y la probabilidad de salir del paro se analiza en dos horizontes temporales (un trimestre después y cuatro trimestres después). La variable de prestaciones es similar a la utilizada en el trabajo comentado previamente. Los resultados indican que para el período 19921998 la variable de prestaciones afecta negativamente a la probabilidad de salida en un horizonte de un trimestre en el mismo sentido encontrado por él en 1998 pero mucho menos (con el primer método) o nada (con el segundo método) en un horizonte de un año. Los resultados son similares pero incluso más nítidos para el período 1999-2003, ya que en este caso ninguna de las categorías de parados que cobran prestaciones muestra una probabilidad de salida hacia el empleo significativamente menor que el grupo de referencia (quienes no cobran y la antigüedad en su último empleo fue inferior a un año) con ambos métodos de identificación.

Finalmente, en una de sus últimas publicaciones en $2010^{12}$, utilizando la EPA enlazada para el período 1992-2004, obtiene resultados similares a los anteriores en el sentido de que la percepción de prestaciones sólo influye negativamente en la salida hacia el empleo temporal (en el caso de las mujeres) y, sobre todo, hacia el autoempleo, pero no hacia el empleo indefinido. Además, la percepción de prestaciones inhibe la transición hacia la inactividad tanto para varones como para mujeres.

En cuanto a la tercera línea de investigación, debe mencionarse que Luis Toharia colaboró intensamente en el diseño y elaboración de la MCVL y fue uno de los primeros en utilizar esta base de datos para la investigación. A pesar de la complejidad del uso de la misma cuando se trata de construir información longitudinal para varios años, Luis Toharia se volcó en ello con el objetivo de analizar cuestiones relacionadas con las políticas de empleo y de protección social. En el caso del SPD, a partir de un estudio financiado por el Ministerio de Trabajo ${ }^{13}$ que dio lugar a un artículo publicado en 2010 (con los datos de la MCVL correspondientes a los años 2004 a 2007) ${ }^{14}$, Luis Toharia presenta nueva evidencia sobre la influencia de las prestaciones por desempleo en el proceso de salida desde el paro hacia la ocupación. No utiliza un sólo año, como la mayoría de los estudios que utilizan la MCVL, sino que Luis Toharia prefiere utilizar todas las olas disponibles de esta base de datos para poder seguir de forma más fiable la vida laboral de los individuos que finalizan un empleo y

12 Arranz, García-Serrano y Toharia (2010).

13 Toharia et al. (2009).

14 Toharia et al. (2010). 
seguirles en el tiempo para conocer si reciben prestaciones o no, y por cuanto tiempo, y si encuentran un empleo estable o no, y por cuanto tiempo.

Los resultados indican que quienes reciben prestaciones por desempleo muestran una tasa de salida hacia el empleo menor y una duración de los episodios de paro mayor que quienes no reciben prestaciones. Cuando los perceptores son clasificados en distintos grupos atendiendo al tipo de prestación y a la duración potencial de la misma (aproximada por la duración de su empleo anterior), lo que encuentra es que este efecto desincentivador se concentra en determinados colectivos: quienes reciben prestaciones asistenciales y contributivas largas y, sobre todo, los perceptores del subsidio de mayores de 52. Además, los no perceptores que realizan estas transiciones más rápidas también presentan duraciones de los empleos más cortas, lo que significa que también vuelven al paro rápidamente, por lo que el supuesto efecto desincentivador de la salida del paro hacia el empleo estaría compensado por una mayor permanencia en el empleo y una menor probabilidad de salida del empleo hacia el paro de los beneficiarios de prestaciones por desempleo. Esto implicaría la existencia de un segmento de trabajadores que entran y salen del empleo muy deprisa y que quedan al margen del sistema de protección social, y otro segmento de trabajadores más estables tanto en el empleo como en el desempleo, lo cual estaría relacionado con el fenómeno de la recurrencia detectado en estudios anteriores.

\section{Las prestaciones por desempleo y la tasa de cobertura}

En 1997 Luis Toharia publicó uno de sus artículos pioneros sobre las características básicas de $\mathrm{SPD}^{15}$, a partir del estudio realizado para la Fundación Empresa Pública citado más arriba, habiendo servido de guía a investigadores posteriores para conocer el sistema desde su inicio con la Ley Básica de Empleo de 1980 hasta los cambios introducidos en la década de los noventa (reformas de 1989-1992 y 1993), su intensidad protectora comparada con el resto de países europeos y el grado de cobertura.

A este respecto, discute la forma de calcular la tasa de sustitución, describiendo la proporción de la población que disfruta de prestaciones, utilizando varias bases de datos y calculando la tasa de cobertura según diferentes características de los parados. Sus discusiones sobre la tasa de

15 Toharia (1997). 
cobertura desarrolladas en este trabajo y en otros posteriores provocaron modificaciones en el cálculo de la misma con el transcurso de los años. Como explica Luis Toharia en uno de los últimos proyectos en que participó $^{16}$, la tasa de cobertura (cociente entre el número de beneficiarios de prestaciones y el número de parados registrados) resultaba incoherente debido a que las exclusiones del paro registrado, cada vez más intensas, no se acompañaban de las exclusiones correspondientes en el número de beneficiarios: se reducía el colectivo de los teóricamente protegibles, sin alterar el colectivo de los teóricamente protegidos. En esta situación, el Servicio Público de Empleo Estatal decidió suspender la publicación de la tasa de cobertura en el avance de septiembre de 2008. Esto llevó a la publicación de un "nuevo indicador" de cobertura definido como el cociente entre el total de beneficiarios de prestaciones, por una parte, y la suma de los parados registrados SISPE con experiencia laboral anterior y los beneficiarios de subsidio de eventuales agrarios, por otra parte.

\section{Reflexiones finales}

La evidencia empírica aportada por los estudios que Luis Toharia llevó a cabo en colaboración con otros autores permite señalar varias conclusiones de interés referidas al vínculo entre el sistema de protección y los procesos de búsqueda de empleo y de salida del paro de los trabajadores desempleados.

Con respecto a la búsqueda de empleo, dos resultados clave. El primero es que, aunque el SPD pudiera contener una dosis de ineficiencia provocada por el esperado desincentivo a la búsqueda de empleo que conlleva cualquier sistema de prestaciones, paradójicamente la existencia de esa ineficiencia no se traduce en un incremento de la tasa de paro (al menos por esta vía) sino en una disminución, ya que los perceptores que no buscan son excluidos de las cifras de parados. Dicho de otro modo, la búsqueda de empleo se ve afectada por la percepción de prestaciones pero eso lleva a los individuos sin empleo a ser clasificados como inactivos no como parados.

El segundo resultado es que la percepción de prestaciones por desempleo no parece afectar de forma significativa ni a la búsqueda activa de empleo ni a la intensidad de búsqueda, condicionado a que las personas declaren que realizan actividades de búsqueda. Sin embargo, a la hora de aceptar un empleo con determinadas condiciones, los perceptores de prestaciones parecen mostrar una menor disponibilidad.

16 Toharia et al. (2009). 
En relación con la influencia de las prestaciones por desempleo en la salida del paro, el hallazgo habitual es que los perceptores permanecen en la situación de paro más tiempo que los no perceptores. Sin embargo, los resultados obtenidos por Luis Toharia tienden a matizar este resultado, al menos en dos aspectos. Por una parte, no tiene sentido considerar a todos los perceptores con un colectivo homogéneo, ya que tanto los diferentes tipos de prestaciones como la duración potencial de las mismas generan efectos distintos sobre la salida hacia el empleo. Por otra parte, algunos datos apuntan a que este efecto quizás es más importante a corto a que medio plazo, al estar ligada la mayor propensión de salida a la fuerte rotación existente en el mercado de trabajo. De ser así, las prestaciones por desempleo estarían permitiendo a los perceptores realizar una mejor búsqueda de empleo. Aunque también hay que decir que la relativamente elevada recurrencia del desempleo cobrando prestaciones (es decir, entradas sucesivas en el sistema de protección por desempleo, especialmente en el nivel contributivo) apuntaría a que el sistema se podría estar utilizando de forma perversa en combinación con los contratos de naturaleza temporal.

Por ello, la evaluación de la eficiencia del sistema de prestaciones por desempleo debe realizarse adoptando una perspectiva temporal y de historia laboral más amplia, no examinando simplemente la salida del paro de los individuos. El análisis de este resultado derivado de los trabajos desarrollados por Luis Toharia a lo largo de su carrera representa todo un desafío para los investigadores que nos dedicamos a la economía laboral.

\section{Bibliografía}

Arranz, J.M., García-Serrano, C. y Toharia, L. (2010), "The influence of temporary employment on unemployment exits in a competing risks framework", Journal of Labor Research, 31:67-90.

Cantó, O. y Toharia, L. (2003), "Las prestaciones por desempleo en España: eficiencia y equidad", Hacienda Pública Española, Monografía 2003: 125-156.

Cebrián I., García-Serrano, C., Muro, J., Toharia, L. y Villagómez, E. (1995), "Prestaciones por desempleo, duración y recurrencia del paro", 155-193, en J.J. Dolado y J.F. Jimeno (comps.), Estudios sobre el 
funcionamiento del mercado de trabajo español, Colección Estudios, 13, FEDEA, Madrid.

Cebrián, I., García-Serrano, C., Muro, J., Toharia, L. y Villagómez, E. (1996a), Protección social y acceso al empleo, Colección Estudios, 34, Consejo Económico y Social, Madrid.

Cebrián I., García-Serrano, C., Muro, J., Toharia, L. y Villagómez, E. (1996b), "The incidence of unemployment compensation on unemployment duration: evidence from Spain”, Labour, 10(2): 239-267.

García Mainar, I. y Toharia, L. (2000), "Prestaciones por desempleo y búsqueda de empleo", Revista de Economía Aplicada, 23: 5-33.

Garrido, L. y Toharia, L. (2003), "What does it take to be (counted as) unemployed? The case of Spain”, Labour Economics, 11(4): 507-523.

Toharia, L. (1997), "El sistema español de protección por desempleo", Papeles de Economía Española, 72: 192-213.

Toharia, L. (dir.) (1998), El mercado de trabajo en España, McGraw Hill, Madrid.

Toharia, L. y Fina, L. (1987), Las causas del paro en España: un punto de vista estructural, Madrid, Fundación IESA.

Toharia, L., Pérez Infante, J.I. y Prudencio, C.A. (2006), La ocupabilidad de los parados registrados y la tasa de cobertura de las prestaciones por desempleo, Informe para el Servicio Público de Empleo Estatal.

Toharia, L., Arranz, J.M., García-Serrano, C. y Hernanz, V. (2009), El sistema español de protección por desempleo: equidad, eficiencia y perspectivas, FIPROS 2008/26, Ministerio de Trabajo e Inmigración.

Toharia, L., Arranz, J.M., García-Serrano, C. y Hernanz, V. (2010), "El sistema de protección por desempleo y la salida del paro", Papeles de Economía Española, 124: 230-246. 